\title{
Origins and organization of the NHLBI State of the Science Workshop: Generating a national blueprint for future research on factor VIII inhibitors
}

\author{
Denise E. Sabatino $^{1}$ (D) | Steven W. Pipe ${ }^{2}$ (D) | Diane J. Nugent ${ }^{3} \mid$ J. Michael Soucie ${ }^{4^{*}}$ | \\ W. Craig Hooper ${ }^{4}$ | W. Keith Hoots ${ }^{5}$ | Donna M. DiMichele ${ }^{5}$
}

${ }^{1}$ Perelman School of Medicine,

The Children's Hospital of

Philadelphia, University of Pennsylvania,

Philadelphia, Pennsylvania

${ }^{2}$ University of Michigan, Ann Arbor,

Michigan

${ }^{3}$ Children's Hospital of Orange

County, University of California at Irvine,

Irvine, California

${ }^{4}$ Division of Blood Disorders, Centers for Disease Control and Prevention, Atlanta, Georgia

${ }^{5}$ Division of Blood Diseases and Resources, National Heart, Lung and Blood Institute, National Institutes of Health,

Bethesda, Maryland

Correspondence

Denise E. Sabatino, The University of Pennsylvania, The Children's Hospital of Philadelphia, Philadelphia, PA.

Email: dsabatin@pennmedicine.upenn.edu
Introduction: The major complication of protein replacement therapy for haemophilia $A$ is the development of anti-FVIII antibodies or inhibitors that occur in $25 \%-30 \%$ of persons with severe haemophilia A. Alternative therapeutics such as bypassing agents or immune tolerance induction protocols have additional challenges and are not always effective.

Aim: Assemble a National Heart, Lung and Blood Institute (NHLBI) State of the Science (SOS) Workshop to generate a national blueprint for research on inhibitors to solve the problem of FVIII immunogenicity.

Methods: An Executive Steering Committee was formed in October 2017 to establish the scientific focus and Scientific Working Groups for the SOS Workshop in May 2018. Four working groups were assembled to address scientific priorities in basic, translational and clinical research on inhibitors.

Results: Working Group 1 was charged with determining the scientific priorities for clinical trials to include the integration of non-intravenous, non-factor therapeutics including gene therapy into the standard of care for people with haemophilia A with inhibitors. Working Group 2 established the scientific priorities for 21st-century data science and biospecimen collection for observational inhibitor cohort studies. The scientific priorities for acquiring an actionable understanding of FVIII immunogenicity and the immunology of the host response and FVIII tolerance were developed by Working Group 3. Working Group 4 designed prospective pregnancy/birth cohorts to study FVIII immunogenicity, inhibitor development and eradication.

Conclusion: The NHLBI SOS Workshop generated a focused summary of scientific priorities and implementation strategies to overcome the challenges of eradicating and preventing inhibitors in haemophilia $\mathrm{A}$.

KEYWORDS

factor VIII, haemophilia, immune response, inhibitor formation 


\section{1 | INTRODUCTION: HAEMOPHILIA AND THE DEVELOPMENT OF INHIBITORS TO FACTOR VIII}

Haemophilia $A$ is an X-linked bleeding disorder that is due to a deficiency in coagulation factor VIII (FVIII). Haemophilia occurs in about 1 in 5000 male births, $80 \%$ of whom are affected with haemophilia A which translates to approximately 16000 persons with haemophilia A (PWHA) in the United States. ${ }^{1}$ PWHA with the severe form of the disorder ( $<1 \%$ of normal plasma FVIII activity) present with frequent spontaneous bleeding episodes that occur primarily in the joints and soft tissues. ${ }^{2}$

The current treatment for haemophilia is protein replacement therapy with plasma-derived or recombinant factor VIII proteins that are given on-demand in response to bleeds or prophylactically with the goal of preventing bleeding episodes. ${ }^{3}$ While this therapy has transformed the care of PWHA, the major complication of replacement therapy is the development of neutralizing alloantibodies to the FVIII protein, termed inhibitors, which at high titre ( $\geq 5$ Bethesda Units) render the therapy ineffective. ${ }^{4}$ This is most significant in severe haemophilia A where $25 \%-30 \%$ of people develop clinically significant anti-FVIII antibodies at a median age of 15 months and after a median of 14 exposures to factor VIII. ${ }^{5,6}$ Importantly, the impact of inhibitors on the patient is significant with more frequent hemarthroses, more severe arthropathy, a reduced quality of life and an increased risk of death. ${ }^{7-9}$

Both genetic and environmental risk factors have been associated with inhibitor development. ${ }^{5,6}$ Genetic factors may include the FVIII mutation, the severity of the haemophilia, family history of inhibitors, ethnicity and polymorphisms of immune response genes. Environmental factors may include the FVIII product (plasma-derived vs recombinant), intensity of FVIII exposure, age at the start of treatment and events such as infection, inflammation and surgery. However, an incomplete mechanistic understanding of risk factors interpreted through informative immune biomarkers limits the ability to both accurately predict inhibitor development in an individual child with severe haemophilia $A$ and intervene with timely inhibitor prevention or eradication strategies. ${ }^{10}$

The development of inhibitors to FVIII results in the neutralization or rapid clearance of the FVIII protein that renders the FVIII therapy ineffective. Until recently, the mainstay therapy for bleeding in the presence of an inhibitor has been the use of bypassing agents, activated prothrombin complex concentrates ${ }^{11}$ or recombinant factor VIIa, ${ }^{12}$ to achieve haemostasis. These treatments are associated with additional challenges including reduced efficacy in the treatment or prevention of haemorrhage compared to FVIII products in those without an inhibitor and the inability to accurately predict the bleeding response. ${ }^{13-15}$ An alternative approach for treating these patients is to attempt to induce immune tolerance to the FVIII protein through immune tolerance induction (ITI) protocols. ${ }^{16}$ Several ITI regimens have been developed that use a range of FVIII dosing regimens, occasionally accompanied by immunomodulation. ${ }^{17,18}$ However, the studies have not yielded a consensus on a practice approach for ITI in part due to the number of factors that influence the success of ITI. In addition, the economic burden of this treatment is significant since the annual cost in the United States increases between $\$ 150000$ and $\$ 200000$ without inhibitors to almost $\$ 1000000$ with an inhibitor. ${ }^{19}$

Novel non-factor therapeutics to treat haemophilia in the presence of inhibitors are on the horizon. ${ }^{20-22}$ Emicizumab, a humanized bispecific monoclonal antibody that mimics the function of FVIII, has recently been approved for use in PWHA with or without FVIII inhibitors. ${ }^{20,23}$ Gene therapy approaches are also in clinical development with phase III clinical trials underway for PWHA without inhibitors. ${ }^{24}$ This single dose treatment may also provide a promising new treatment for PWHA and inhibitors that have the potential to be a lifelong therapy. While these new therapies may change the approach to treating patients, national and international data gathered from harmonized and standardized observational cohorts and innovatively designed clinical trials will be required to integrate them into the standard of care for PWHA with inhibitors. ${ }^{25,26}$

\section{ORIGINS OF THE STATE OF THE SCIENCE WORKSHOP}

Within the United States, it is estimated that there are at least 1000 individuals with a factor VIII inhibitor. ${ }^{27}$ The Centers for Disease Control and Prevention (CDC)'s Division of Blood Disorders (DBD) have been committed to the goal of reducing the occurrence of inhibitors, the most significant and costly complication affecting persons with haemophilia today. Following a multi-stakeholder summit in 2012, the CDC facilitated a national integrated inhibitor surveillance programme through a cooperative agreement with the US Hemophilia Treatment Center Network (USHTCN) and the American Thrombosis and Hemostasis Network (ATHN) to collect information about key aspects of inhibitor development, treatment and outcomes and established the DBD Reference Laboratory to develop the methodology required for centralized sensitive and specific inhibitor testing. ${ }^{28}$ A second multi-stakeholder summit in 2017 included an objective to explore the need for a national, prioritized inhibitor scientific agenda and the blueprint for its coordinated implementation. The rationale for this coordinated effort included:

- Subjects needed for studies in this area (primarily previously untreated patients) are a precious resource, and efforts should be made to coordinate studies so that the maximum benefit can be obtained from each study subject.

- Oversight is needed to assure that only the most promising science is performed and funding for the studies should be adequate to cover the costs of obtaining high-quality data.

- Multifaceted education and informational activities must be directed to the patient community well in advance of upcoming trials and are required to stimulate interest and participation. 
TABLE 1 Working Group 1: scientific priorities and innovative implementation strategies for FVIII inhibitor clinical trials

$\begin{array}{ll}\text { Co-chairs } & \text { Margaret Ragni, MD, MPH and Lindsey George, MD } \\ \text { Charge } & \text { The design of investigator-initiated clinical trials to determine optimal integration } \\ \text { of non-IV, non-factor therapeutics, including gene therapy, into the standard of } \\ \text { care for FVIII inhibitor patients }\end{array}$

Co-chairs Barbara Konkle, MD and Mike Recht, MD, PhD

Charge The design of and supportive infrastructure for prospective longitudinal cohorts to ascertain comparative short and medium-term outcomes from the incorporation, or not, of non-intravenous, non-factor novel therapeutics, including gene therapy, into the standard of care for FVIII inhibitors

Goals

- Incorporation of standard measures for prioritized outcomes

- Incorporation of patient-reported outcomes (PROs)

- Models for direct data transfer from electronic medical records (EMRs)

- Potential for the CDC surveillance databases and central laboratory to serve as a platform for launching prospective clinical trials

- Streamlined data sharing policies for individual patient level data

- Challenges associated with developing and maintaining data repositories and biobanks in rare diseases; resources and partnerships required to facilitate and maintain these repositories

- Optimizing private-public partnerships to fund clinical research

- Engage the patient community in observational cohort studies

- Training opportunities in epidemiology and data science
- Development of a multidisciplinary group is required to develop and implement an integrated scientific and public health agenda as well as to establish the infrastructure within which the essential science can be conducted.

- Representatives from a wide variety of disciplines should be included to facilitate the generation of new ideas and approaches.

- Agreement from the bleeding disorder community to proceed with regimented, cooperative, appropriately vetted studies must be secured.

Following this CDC summit, the Medical and Scientific Advisory Council (MASAC) to the National Hemophilia Foundation formed the MASAC Inhibitor Prevention and Eradication Working Group in March 2017 with a charter that included a mandate to engage the haemophilia community in the development of a national scientific agenda that would ensure the coordinated future conduct of the most efficient and impactful research. The MASAC Inhibitor Prevention and Eradication Working Group, in collaboration with the Division of Blood Diseases and Resources of the National Heart Lung and Blood Institute (NHLBI)/National Institutes of Health (NIH), developed the concept for The NHLBI State of the Science (SOS) Workshop. The goal of the workshop was to solicit input from the haemophilia community as well as from experts from outside the field into the development of a coordinated US-based blueprint for future basic, translational and clinical research focused on FVIII immunogenicity and factor VIII inhibitor prevention/eradication.

\section{3 | ORGANIZATION OF THE STATE OF THE SCIENCE WORKSHOP}

The efforts to assemble the SOS Workshop began in October 2017. The Executive Steering Committee, constituted by the authors of this manuscript, was established and given the mandate to establish the scientific focus for as well as the leadership and membership of the Scientific Working Groups, to oversee the pre-SOS Working Group deliberations and to develop the SOS Workshop agenda. Four scientific priorities were identified across the spectrum of basic, translational and clinical research, and the working groups were organized around these specific topics (Tables 1-4). Working group members were deliberately assembled (1) to ensure that diverse perspectives from across the national and international haemophilia community informed each working group's deliberations, and (2) to maximally stimulate scientific thought beyond the current principles and approaches with relevant complimentary 


$\begin{array}{ll}\text { Co-chairs } & \text { Shannon Meeks, MD and Roland Herzog, PhD } \\ \text { Charge } & \text { Basic and translational research to elucidate an actionable understanding of FVIII } \\ \text { immunogenicity and the immunology of both the host immune response and } \\ \text { tolerance to inform predictive models for inhibitor development and novel } \\ \text { therapeutic targets } \\ \text { - Application of novel ideas, technologies and cross-disciplinary science to these } \\ \text { - studies } \\ \text { - Consideration of the role of host cell expression of FVIII/VWF in immuno- } \\ \text { - } \text { - Idenicity as well as gene therapy as a model for FVIII immune tolerance } \\ \text { - expression and peptide generation } \\ \text { - Human biospecimens required to further research } \\ \text { - Challenges associated with and successful models for conducting cross-disci- } \\ \text { plinary science as well as resources and partners required for success } \\ \text { - Optimization of private-public partnerships in basic/translational research } \\ \text { - Training models and opportunities engendered by novel cross-disciplinary } \\ \text { science }\end{array}$

Co-chairs Deborah Brown, MD and Jill Johnsen, MD

Charge The design of prospective pregnancy/birth longitudinal cohorts that leverage multi-"omics" science, existing phenotypic data and in silico protein modelling to study FVIII immunogenicity, inhibitor development and eradication

Goals

- Design data capture and mechanistic studies, based on translational scientific priorities required to build precision (personalized) medicine-based

- Clinical decision-making algorithms that can be applied across the lifespan to either avoid or provoke clinical phenotype for the purpose of diagnosis and/or appropriate time-sensitive intervention, include the design of potential antenatal/neonatal interventions

- Address challenges associated with, and successful models for building lifespan/intergenerational cohorts, as well as resources and partners required

- For success, including unique challenges in sample procurement and banking

- Optimization of private-public partnerships to fund longitudinal cohorts

- Engage the patient community in longitudinal cohort participation

- Training models and opportunities engendered by novel cross-disciplinary science
TAB LE 3 Working Group 3: scientific priorities and implementation strategies for acquiring an actionable understanding of FVIII immunogenicity and the immunology of both the host immune response and tolerance
TABLE 4 Working Group 4: design of pregnancy/birth longitudinal cohorts that leverage 'omics', existing phenotypic data, and in silico modelling to study FVIII immunogenicity, as well as inhibitor development and eradication expertise from outside the field (Tables S1-S4). Expertise from broad scientific areas was assembled to include FVIII biochemistry, immunology, "omics," gene therapy, maternal and foetal biology, epidemiology, and computational biology. The working groups also brought together extensive knowledge in clinical trial design, biostatistics, human subjects research, biobanking, data sharing and ethics. The pharmaceutical industry was represented on each working group. The haemophilia community was represented by $\mathrm{PWH}$, patient advocacy groups and members of the haemophilia treatment centres (HTC). Financial conflicts of interest were declared to the Working Group Chairs, as well as to the NHLBI, and were presented at the SOS Workshop.

Once the working groups were formed, their deliberations began in January 2018 and continued in the form of bi-weekly discussions to determine the scientific priorities in each area. In addition, there was significant crosstalk among the working groups. On 15 and 16 May 2018, the culmination of these efforts was presented as a draft of research priorities and implementation strategies from each working group at the NHLBI State of the Science Workshop on FVIII Inhibitors at the National Institutes of Health in Bethesda, MD. Input from the wider community was solicited through both plenary and working group-specific breakout session discussions. Keynote speakers selected from outside the haemophilia community provided insights on topics related to each of the four working group scientific priorities. These topics included clinical trial design in the age of personalized medicine (Nicholas Schork, PhD, J. Craig Venter Institute, University of California, San Diego), learning health systems for assembling clinical research data (Charles Bailey, MD, PhD, The Children's Hospital of Philadelphia), the development of patient registries and cohorts in rare diseases (Jennifer Mulle, PhD, MHS, Emory University) and the use of the knowledge of immune pathways to reduce protein immunogenicity (Elizabeth Mellins, MD, Lucile Salter Packard Children's Hospital, Stanford University). The 2-day workshop was attended by more than 200 participants from 29 states and nine countries: 54\% represented academia and HTCs; 
$18 \%$ were scientists from the federal government; $20 \%$ were from the pharmaceutical industry; and 8\% represented patient advocacy groups. A videocast of the proceedings was archived at the NHLBI and is available for public viewing. ${ }^{29,30}$

\section{1 | Working Group 1: scientific priorities and innovative implementation strategies for FVIII inhibitor clinical trials}

Co-chaired by Margaret Ragni, MD, MPH and Lindsey George, MD, Working Group 1 was charged with ascertaining the scientific priorities for investigator-initiated clinical trials to include the optimal integration of non-intravenous, non-factor novel therapeutics including gene therapy into the standard of care for PWHA with inhibitors (Table 1). The expanded goals established for this group focused on the optimization of both clinical trial design and national infrastructure requirements to increase the feasibility of a national clinical trial agenda. ${ }^{31}$

\section{2 | Working Group 2: scientific priorities and strategies for 21st-century data and specimen collection and observational FVIII inhibitor cohort studies}

Working Group 2 was co-chaired by Barbara Konkle, MD and Mike Recht, MD, PhD. This group was charged with establishing the scientific priorities for 21st-century data science and biospecimen collection. Design parameters include prospective cohorts to ascertain comparative short and medium-term outcomes from the incorporation, or not, of non-intravenous, non-factor novel therapeutics, including gene therapy, into the standard of care for FVIII inhibitors (Table 2). The goals for this group focused on overcoming the challenges associated with developing and maintaining data and biospecimen repositories and included deliberations about the infrastructure requirements for creating a data and biospecimen repository as well as strategies for implementation of a platform to establish this cohort. ${ }^{32}$

\section{3 | Working Group 3: scientific priorities and implementation strategies for acquiring an actionable understanding of FVIII immunogenicity and the immunology of both the host immune response and tolerance}

Co-chaired by Shannon Meeks, MD and Roland Herzog, PhD, Working Group 3 was charged with developing the scientific priorities for acquiring an actionable understanding of FVIII immunogenicity and the immunology of the host response and FVIII tolerance. Such data can inform predictive models for inhibitor development and novel therapeutic targets (Table 3 ). The goals of this group focused on the application of novel ideas, technologies and cross-disciplinary science to these studies. ${ }^{33}$

\section{4 | Working Group 4: design of pregnancy/birth longitudinal cohorts that leverage omics, existing phenotypic data and in silico modelling to study FVIII immunogenicity, as well as inhibitor development and eradication}

Working Group 4, led by Deborah Brown, MD and Jill Johnsen, MD, was charged with designing of prospective pregnancy/birth cohorts that leverage multi-"omics" science, existing phenotype data and in silico protein modelling to study FVIII immunogenicity, inhibitor development and eradication (Table 4). The goals established for this group are also summarized in Table 4. These were primarily focused on the design of data capture and mechanistic studies, based on translational scientific priorities, required to build a personalized medicine-based clinical decision-making algorithm. Such an algorithm can be applied across the lifespan to either avoid or provoke the clinical phenotype for the purpose of diagnosis and/or appropriate time-sensitive intervention. ${ }^{34}$

\section{CONCLUSION}

The State of the Science Workshop assembled the key stakeholders in the challenge to eradicate and prevent inhibitors: the patients, clinicians, researchers, federal government and industry. Through the commitments of the Executive Steering Committee, the Working Group Chairs and all the members of the Working Groups, the many hours of deliberations lead to a focused summary of scientific priorities and implementation strategies to methodically tackle the challenges of understanding the immune response to factor VIII and reaching the goal of eradicating and preventing inhibitors. ${ }^{35}$

\section{DISCLOSURES}

D. Nugent is a member of the advisory board for NovoNordisk factor XIII and Bayer. All other authors have no conflicts of interest to report.

ORCID

Denise E. Sabatino (iD https://orcid.org/0000-0002-6116-5453

Steven W. Pipe (D) https://orcid.org/0000-0003-2558-2089

\section{REFERENCES}

1. Soucie JM, Evatt B, Jackson D. Occurrence of hemophilia in the United States. The hemophilia surveillance system project investigators. Am J Hematol. 1998;59(4):288-294.

2. Carcao M, Moorehead P, Lillicrap D. Hemophilia A and B. In: Hoffman R, Benz EJ, Silberstein LE, et al., eds. Hematology: Basic Principles and Practice, 7 edn. Philadelphia, PA: Elsevier; 2017:2001-2022. 
3. Manco-Johnson MJ, Abshire TC, Shapiro AD, et al. Prophylaxis versus episodic treatment to prevent joint disease in boys with severe hemophilia. N Engl J Med. 2007;357(6):535-544.

4. Blanchette VS, Key NS, Ljung LR, Manco-Johnson MJ, van den Berg HM, Srivastava A. Definitions in hemophilia: communication from the SSC of the ISTH. J Thromb Haemost. 2014;12(11):1935-1939.

5. Gouw SC, van den Berg HM, Fischer K, et al. Intensity of factor VIII treatment and inhibitor development in children with severe hemophilia A: the RODIN study. Blood. 2013;121(20):4046-4055.

6. Gouw Sc, van der Bom Jg, Marijke van den Berg H. Treatment-related risk factors of inhibitor development in previously untreated patients with hemophilia A: the CANAL cohort study. Blood. 2007;109(11):4648-4654.

7. Scalone L, Mantovani LG, Mannucci PM, Gringeri A, COCIS Study Investigators. Quality of life is associated to the orthopaedic status in haemophilic patients with inhibitors. Haemophilia. 2006;12(2):154-162.

8. Gringeri A, Mantovani LG, Scalone L, Mannucci PM, COCIS Study Group. Cost of care and quality of life for patients with hemophilia complicated by inhibitors: the COCIS Study Group. Blood. 2003;102(7):2358-2363.

9. Walsh CE, Soucie JM, Miller $\mathrm{CH}$, United States Hemophilia Treatment Center Network. Impact of inhibitors on hemophilia A mortality in the United States. Am J Hematol. 2015;90(5):400-405.

10. Hashemi SM, Fischer K, Moons K, van den Berg HM. Improved prediction of inhibitor development in previously untreated patients with severe haemophilia A. Haemophilia. 2015;21(2):227-233.

11. Lusher JM, Shapiro SS, Palascak JE, Rao AV, Levine PH, Blatt PM. Efficacy of prothrombin-complex concentrates in hemophiliacs with antibodies to factor VIII: a multicenter therapeutic trial. N Engl J Med. 1980;303(8):421-425.

12. Hedner U, Kisiel W. Use of human factor VIla in the treatment of two hemophilia A patients with high-titer inhibitors. J Clin Invest. 1983;71(6):1836-1841.

13. Astermark J, Donfield SM, DiMichele DM, et al. A randomized comparison of bypassing agents in hemophilia complicated by an inhibitor: the FEIBA NovoSeven Comparative (FENOC) study. Blood. 2007;109(2):546-551.

14. Konkle BA, Ebbesen LS, Erhardtsen E, et al. Randomized, prospective clinical trial of recombinant factor VIla for secondary prophylaxis in hemophilia patients with inhibitors. J Thromb Haemost. 2007;5(9):1904-1913.

15. Leissinger C, Gringeri A, Antmen B, et al. Anti-inhibitor coagulant complex prophylaxis in hemophilia with inhibitors. N Engl J Med. 2011;365(18):1684-1692.

16. Schep SJ, Schutgens R, Fischer K, Boes ML. Review of immune tolerance induction in hemophilia A. Blood Rev. 2018;32(4):326-338.

17. DiMichele DM. Immune tolerance in haemophilia: the long journey to the fork in the road. Br J Haematol. 2012;159(2):123-134.

18. Valentino LA, Kempton Cl, Kruse-Jarres R, Mathew P, Meeks SI, Reiss UM. US Guidelines for immune tolerance induction in patients with haemophilia a and inhibitors. Haemophilia. 2015;21(5):559-567.

19. Zhou Z-Y, Koerper MA, Johnson KA, et al. Burden of illness: direct and indirect costs among persons with hemophilia $A$ in the United States. J Med Econ. 2015;18(6):457-465.

20. Uchida N, Sambe T, Yoneyama K, et al. A first-in-human phase 1 study of ACE910, a novel factor VIII-mimetic bispecific antibody, in healthy subjects. Blood. 2016;127(13):1633-1641.

21. Pasi KJ, Rangarajan S, Georgiev P, et al. Targeting of antithrombin in hemophilia A or B with RNAi therapy. N Engl J Med. 2017;377(9):819-828.

22. Eichler $\mathrm{H}$, Angchaisuksiri $\mathrm{P}$, Kavakli $\mathrm{K}$, et al. A randomized trial of safety, pharmacokinetics and pharmacodynamics of concizumab in people with hemophilia A. J Thromb Haemost. 2018;16(11):2184-2195.
23. Oldenburg J, Mahlangu JN, Bujan W, et al. The effect of emicizumab prophylaxis on health-related outcomes in persons with haemophilia A with inhibitors: HAVEN 1 study. Haemophilia. 2019;25(1):33-44.

24. George LA. Hemophilia gene therapy comes of age. Blood Adv. 2017;1(26):2591-2599.

25. Young G. Implementing emicizumab in hemophilia inhibitor management: emicizumab should be prescribed after tolerance. Blood Adv. 2018;2(20):2780-2782.

26. Le Quellec S, Négrier C. Emicizumab should be prescribed independent of immune tolerance induction. Blood Adv. 2018;2(20):2783-2786.

27. https://www.cdc.gov/ncbddd/hemophilia/inhibitors.html. Accessed February 5, 2019.

28. Soucie JM, Miller CH, Kelly FM, et al. National surveillance for hemophilia inhibitors in the United States: summary report of an expert meeting. Am J Hematol. 2014;89:621-625.

29. State of the science inhibitors workshop: FVIII inhibitors: generating a national blueprint for future research (Day 1). https://videocast. nih.gov/Summary.asp?Live=27769\&bhcp=1. Accessed February 5, 2019.

30. State of the science inhibitors workshop: FVIII inhibitors: generating a national blueprint for future research (Day 2). https://videocast. nih.gov/summary.asp?Live $=27775 \& b h c p=1$. Accessed February 5, 2019.

31. Ragni M, George L; on behalf of the Members of Working Group 1. The national blueprint for future factor VIII inhibitor clinical trials: NHLBI State of the Science (SOS) Workshop on factor VIII inhibitors. Haemophilia. 2019;25(4):581-589.

32. Konkle BA, Recht M; on behalf of the Members of Working Group 2. The national blueprint for 21st century data and specimen collection and observational cohort studies: NHLBI State of the Science (SOS) Workshop on factor VIII inhibitors. Haemophilia. 2019;25(4):590-594.

33. Meeks SL, Herzog RW; on behalf of the Members of Working Group 3. The national blueprint for future basic and translational research to understand factor VIII immunogenicity: NHLBI State of the Science (SOS) Workshop on factor VIII inhibitors. Haemophilia. 2019;25(4):595-602.

34. Johnsen JM, Brown DL; on behalf of the Members of Working Group 4. The national blueprint for pregnancy/birth longitudinal cohorts to study factor VIII immunogenicity: NHLBI State of the Science (SOS) Workshop on factor VIII inhibitors. Haemophilia. 2019;25(4):603-609.

35. Pipe SW, Sabatino DE, Nugent DJ, et al. Executive summary of the NHLBI State of the Science (SOS) Workshop: Overview and next steps in generating a national blueprint for future research on factor VIII inhibitors. Haemophilia. 2019;25(4):610-615.

\section{SUPPORTING INFORMATION}

Additional supporting information may be found online in the Supporting Information section at the end of the article.

How to cite this article: Sabatino DE, Pipe SW, Nugent DJ, et al. Origins and organization of the NHLBI State of the Science Workshop: Generating a national blueprint for future research on factor VIII inhibitors. Haemophilia. 2019;25:575-580. https://doi.org/10.1111/hae.13737 